\title{
REVISÃO
}

\section{SUPLEMENTAÇÃO DE VITAMINAS NA PREVENÇÃO DE CÂNCER}

\section{VITAMIN SUPPLEMENTATION IN CANCER CHEMOPREVENTION}

Cyntia Rosa de Melo SILVA'

Maria Margareth Veloso NAVES'

RESUMO

Este trabalho constitui uma revisão de dados epidemiológicos sobre a relação entre o consumo de vitaminas e prevenção de câncer. Estudos observacionais evidenciam uma associação inversa consistente entre a ingestão de frutas e hortaliças e risco de câncer, e um efeito protetor das vitaminas C, E e dos carotenóides contra certos tipos de câncer. Resultados de estudos de intervenção, na prevenção primária de câncer, são contraditórios, sendo que em dois desses estudos o $\beta$-caroteno, suplementado em doses consideradas farmacológicas, isolado ou associado à vitamina $E$ ou à vitamina A, mostrou-se deletério para tabagistas. Ao contrário, a ingestão de doses mais fisiológicas de antioxidantes parece ser mais eficaz, sendo que a ação sinérgica dessas substâncias na quimioprevenção de câncer já foi demonstrada tanto em modelo in vivo de carcinogênese quanto em estudo epidemiológico de intervenção. Como estratégia de prevenção de câncer preconiza-se o consumo de dietas ricas em frutas e hortaliças, que aportem cerca de $150 \mathrm{mg}$ de vitamina $\mathrm{C}, 30 \mathrm{mg}$ de vitamina $E$ e $4 \mathrm{mg}$ de carotenóides. A ingestão de quantidades mais elevadas, na forma de suplementos, não está recomendada.

Termos de indexação: epidemiologia nutricional, quimioprevenção, vitaminas, neoplasias, antioxidantes.

\begin{abstract}
This work is a review of epidemiologic data about the relationship between vitamin intake and cancer prevention. Observational studies have strongly suggested an inverse association between fruit and vegetable intake and cancer risk, and a protector effect of $C$ and $E$ vitamins and carotenoids against some kinds of cancer. In intervention trials $\beta$-carotene was found to be deleterious to smokers, when supplemented in pharmacological doses isolated or associated with vitamin $E$ or vitamin $A$. On the other hand, the consumption of antioxidants in physiological amounts may be effective against cancer, and this synergic action was observed in both in vivo model of carcinogenesis and epidemiologic intervention trial. It has been commended a generous daily consumption of fruits and vegetables, with amounts of $C$ and $E$ vitamins and carotenoids nearly to 150, 30, and $4 \mathrm{mg}$, respectively, and the vitamin supplements are not recommended as chemopreventive agents against cancer
\end{abstract}

Index terms: nutritional epidemiology, chemoprevention, vitamins, neoplasms, antioxidants.

(1) Faculdade de Nutrição, Universidade Federal de Goiás. Rua 227, s/n, Quadra 68, Setor Universitário, 74605-080, Goiânia, GO, Brasil. Fone (0xx62) 202-2553/209-6175, Fax (0xx62) 202-1748. Correspondência para/Correspondence to: M.M.V. NAVES. 


\section{INTRODUÇÃO}

As vitaminas são compostos orgânicos que variam amplamente quanto à estrutura química e atividade biológica, podendo funcionar tanto como co-fatores de enzimas em diferentes reações bioquímicas, quanto como antioxidantes/oxidantes, modulando o balanço oxidativo, e até mesmo como hormônios, regulando a expressão gênica (Olson,1994). Sendo assim, discute-se atualmente a eficácia da ingestão de quantidades elevadas de vitaminas para atender demandas além das suas funções nutricionais, no sentido de prevenção de doenças crônicas tais como as doenças cardiovasculares e o câncer (Olson,1994, Hathcock, 1997).

Há cerca de duas décadas se tem investigado a relação entre o consumo de vitaminas e de carotenóides (independente de sua atividade pró-vitamínica A) e a gênese do câncer, em função de achados promissores de estudos epidemiológicos observacionais (Byers \& Perry, 1992; van Poppel \& van den Berg,1997). Os estudos nesta área são dificultados pelo fato do câncer ser uma síndrome que envolve várias etapas, em geral alinhadas em três estágios definidos como iniciação, promoção e progressão (Pitot \& Dragan,1996). Além disso, o processo de carcinogênese envolve múltiplos fatores determinantes, sendo complicada a tarefa de se isolar um único nutriente como fator causal (Doll,1996).

Estudos epidemiológicos observacionais evidenciam uma associação inversa consistente entre o consumo de frutas e hortaliças ou níveis séricos de $\beta$-caroteno e risco de câncer de pulmão (Ziegler et al.,1996b). Os efeitos protetores observados têm sido atribuídos, em grande parte, ao conteúdo de vitaminas denominadas antioxidantes e de carotenóides desses alimentos (Ziegler et al.,1992; Tavani \& La Vecchia,1995; Ziegler et al.,1996a).

Por outro lado, os resultados disponíveis de quatro estudos epidemiológicos de intervenção objetivando a prevenção primária de câncer e envolvendo a suplementação com doses elevadas de vitaminas são contraditórios (Burri,1997; van Poppel \& van den Berg,1997; Naves,1998). Em dois desses estudos, a suplementação com $\beta$-caroteno (isolado ou associado à vitamina E) (Alpha-Tocopherol...,1994) ou com $\beta$-caroteno mais vitamina A (Omenn et al.,1996), foi prejudicial para indivíduos submetidos a alto risco para câncer de pulmão. Assim, até o momento não se pode recomendar o consumo destas substâncias na forma de suplementos nutricionais, como uma estratégia de prevenção contra o câncer.

Diante do exposto, objetiva-se revisar os resultados dos principais estudos epidemiológicos de intervenção com suplementos de vitaminas A, C e E e carotenóides na prevenção primária de câncer, incluindo uma breve revisão sobre as evidências epidemiológicas que serviram de suporte aos estudos de intervenção, bem como as recomendações mais recentes de ingestão desses nutrientes.

\section{Vitaminas A, C, E e carotenóides}

As vitaminas mais investigadas como substâncias quimiopreventivas são as vitaminas $A$ incluindo os carotenóides e as vitaminas C e E. Sabe-se que, por um lado, as vitaminas $\mathrm{C}$ e $\mathrm{E}$ e os carotenóides funcionam como antioxidantes em sistemas biológicos (Rock et al.,1996; Stahl \& Sies,1997), e por outro, o processo carcinogênico é caracterizado por um estado oxidativo crônico, especialmente na etapa de promoção (Cerutti,1994). Além disso, a fase de iniciação está associada com dano irreversível no material genético da célula, muitas vezes devido ao ataque de radicais livres (Anderson,1996). Desse modo, os nutrientes antioxidantes poderiam reduzir o risco de câncer inibindo danos oxidativos no DNA (Cerutti,1994; Cozzi et al.,1997; Pool-Zobel et al.,1997), sendo portanto considerados como agentes potencialmente quimiopreventivos (Bonne et al.,1990).

As formas químicas e fontes alimentares das vitaminas A, E e C estão apresentadas no Quadro 1, e na Tabela 1 encontram-se as fontes alimentares de nutrientes antioxidantes com suas respectivas quantidades. A vitamina A (retinol) pertence à família de substâncias denominadas retinóides, na qual se inclui o retinol e seus metabólitos naturais (retinal e ácido retinóico), bem como um grande número de análogos sintéticos (Sporn \& Roberts, 1985). A atividade quimiopreventiva dos retinóides observada tanto em modelos experimentais de carcinogênese quanto em alguns tipos de cânceres em humanos, tem sido atribuída à ação do ácido retinóico sobre a expressão de genes envolvidos com a diferenciação e proliferação celulares (Lotan, 1996).

Quadro 1. Formas químicas e fontes alimentares das vitaminas A, E e C.

\begin{tabular}{lll}
\hline Vitamina & Forma química & Fonte alimentar \\
\hline $\mathrm{A}$ & Ésteres de retinila & Leite integral e enriquecido, gema de ovo, fígado, carne \\
& Carotenóides (pró-vitaminas $\mathrm{A}$ ) & Vegetais e frutas de cor verde-escuro e amarelo-alaranjado \\
$\mathrm{E}$ & $\alpha, \beta$ e $\delta$-tocoferol; $\alpha, \beta$ e $\delta$-tocotrienol & Óleos vegetais (milho, girassol, soja, canola), margarina, amendoim, amêndoa, gérmen de trigo \\
$\mathrm{C}$ & Acido ascórbico, ácido dehidroascórbico & Frutas: acerola, caju, goiaba, frutas cítricas \\
& & Vegetais: brócolis, couve, couve-flor, tomate \\
\hline
\end{tabular}


Tabela 1. Fontes alimentares de nutrientes antioxidantes e suas quantidades.

\begin{tabular}{|c|c|c|c|c|}
\hline \multirow{2}{*}{ Fontes } & & Quantidade & \multirow{2}{*}{ Fontes } & \multirow{2}{*}{$\begin{array}{c}\text { Quantidade } \\
\text { (ug/100g) }\end{array}$} \\
\hline & & $(\mathrm{mg} / 100 \mathrm{~g})$ & & \\
\hline \multicolumn{3}{|c|}{ Vitamina $E^{1}$ ( $\alpha$-tocoferol) } & \multicolumn{2}{|r|}{ Carotenóides $^{3}$} \\
\hline & & & Vegetais & \\
\hline Óleo de girassol & & 48,7 & Couve & 26600 \\
\hline Margarinas & & 25,0 & Espinafre & 14300 \\
\hline Amêndoa & & 20,0 & Cenoura & 11760 \\
\hline Gérmen de trigo & & 11,0 & Abóbora & 8400 \\
\hline \multirow[t]{4}{*}{ Amendoim } & & 8,0 & Tomate & 3720 \\
\hline & & & Brócolis & 3100 \\
\hline & Vitamina $C^{2}$ & & Milho & 880 \\
\hline & & & Piqui & 610 \\
\hline \multicolumn{5}{|l|}{ Frutas } \\
\hline \multicolumn{5}{|l|}{ Acerola } \\
\hline Caju & & 1369 & Frutas & \\
\hline Goiaba & & 219 & Buriti & 48880 \\
\hline Laranja (suco) & & 218 & Goiaba vermelha & 6212 \\
\hline Vegetais & & 54 & Pitanga & 1640 \\
\hline Brócolis & & & Manga & 1300 \\
\hline Couve & & 113 & Mamão & 859 \\
\hline Couve-flor & & 92 & Pêssego & 651 \\
\hline \multirow[t]{2}{*}{ Tomate } & & 82 & & \\
\hline & & 23 & & \\
\hline
\end{tabular}

Fontes: ${ }^{(1)}$ Paul \& Southgate (1978); Whitney \& Rolfes (1999).

(2) Azoubel et al. (1998); Pinheiro et al. (2001).

(3) Godoy \& Rodriguez-Amaya (1994), Mangels et al. (1993);

Os carotenóides, por sua vez, constituem uma família de mais de 600 membros já identificados na natureza, sendo que cerca de 50 apresentam atividade pró-vitamínica A, ou seja, podem funcionar como precursores de vitamina A em mamíferos (Olson,1989). Destes, o $\beta$-caroteno é o mais abundante na natureza e é encontrado em vegetais e frutas de cor verde-escuro e amarelo-alaranjado (Mangels et al.,1993; Godoy \& Rodriguez-Amaya,1994; Rock et al.,1996). Algumas das maiores fontes de carotenóides são: cenouras e abóboras ( $\alpha$ - e $\beta$-caroteno); tomates e produtos derivados como extrato, polpa e molhos (licopeno); goiaba vermelha (licopeno) e espinafre (luteína) (Tabela 1). O efeito protetor dos carotenóides, em especial do $\beta$-caroteno, demonstrado em diferentes modelos experimentais in vitro e in vivo, tem sido atribuído mais à uma ação do próprio pigmento do que dos retinóides produzidos a partir do seu metabolismo endógeno (Rock et al.,1996; Naves \& Moreno,1998; Naves \& Moreno, 2000).

O termo vitamina $C$ é uma denominação genérica para todos os compostos que apresentam atividade biológica do ácido ascórbico. Dentre eles, o ácido ascórbico é o mais largamente encontrado nos alimentos e possui maior poder antioxidante (Rock et al.,1996; Stahl \& Sies,1997). Os possíveis efeitos anticarcinogênicos da vitamina $C$ estão relacionados com sua habilidade em detoxicar substâncias carcinogênicas e sua atividade antioxidante (Stahl \& Sies,1997). Além disso, tem-se constatado que a vitamina $\mathrm{C}$ pode inibir a formação de nitrosaminas in vivo a partir de nitratos e nitritos usados como conservantes, sendo portanto adicionada a muitos produtos alimentares industrializados para prevenir a formação desses compostos reconhecidamente carcinogênicos (Kuhn et al.,1991; Bianchi \& Antunes, 1999). As maiores fontes alimentares são as frutas, especialmente a acerola, o caju e a goiaba, e vegetais como brócolis, couve e couve-flor (Tabela 1).

A vitamina E é uma substância lipossolúvel e existente na natureza como tocoferóis e tocotrienóis, em quatro formas diferentes $(\alpha, \beta, \gamma$ e $\delta$ ), sendo o $\alpha$-tocoferol a forma antioxidante mais ativa e amplamente distribuída nos tecidos e no plasma (Niki,1996). A vitamina E constitui o antioxidante lipossolúvel mais efetivo encontrado na natureza, e importante fator de proteção contra a peroxidação lipídica nas membranas celulares e na circulação sangüínea (Rock et al.,1996; Stahl \& Sies, 1997). Os óleos vegetais e as margarinas, além de amêndoas, amendoim e gérmen de trigo, constituem alimentos ricos em vitamina E (Quadro 1 e Tabela 1).

Devido às propriedades químicas das vitaminas $C$ e E e dos carotenóides, substâncias altamente reativas e facilmente oxidadas, podem agir como parte do sistema de defesa antioxidante do organismo humano, quando 
presentes em quantidades fisiológicas e sob determinadas condições intracelulares, como por exemplo, à pressões parciais fisiológicas de oxigênio (Burton \& Ingold,1984; Rock et al.,1996). Ao contrário, em altas concentrações e à pressões parciais de oxigênio elevadas podem funcionar como agentes oxidantes, contribuindo para a formação de radicais livres (Herbert,1996; Palozza,1998; Podmore et al., 1998). Assim, o termo "antioxidante" deve ser utilizado com cautela, sendo proposto por Olson (1996) o termo "moduladores fisiológicos", por ser mais abrangente e incluir, inclusive, os efeitos adversos eventualmente resultantes do uso abusivo dessas substâncias.

\section{Evidências epidemiológicas}

Relatos de estudos epidemiológicos observacionais têm evidenciado que a ingestão de micronutrientes, tais como vitaminas e minerais, pode prevenir alguns tipos de câncer. Dentre os micronutrientes tem-se focalizado o uso da vitamina $\mathrm{A}$ e dos denominados antioxidantes, ou seja, os carotenóides, as vitaminas $\mathrm{C}$ e $\mathrm{E}$, e em alguns casos, o selênio e o zinco (Byers \& Perry,1992; Blumberg,1995; Flagg et al.,1995; Patterson et al.,1997), em estudos tipo caso-controle (retrospectivo) e coorte (prospectivo).

Diversos trabalhos de revisão têm tentado estabelecer, a partir da análise de dados disponíveis na literatura, uma associação entre a ingestão e/ou níveis séricos de nutrientes específicos e o risco de câncer (Knekt,1993; Flagg et al.,1995; Ziegler et al.,1996b). Contudo, conforme mencionado anteriormente, é difícil determinar até que ponto o consumo de um único nutriente pode interferir na quimioprevenção do câncer (Doll,1996). O efeito observado pode ser resultante da ação ou interação de outros componentes dos alimentos de origem vegetal, tais como os compostos polifenólicos e a fibra da dieta (Caragay,1992; Ziegler et al.,1996a), e particularmente a vitamina $C$ e o $\beta$-caroteno podem ser apenas marcadores de outros compostos ativos biologicamente presentes na dieta (Rock et al.,1996; Ziegler et al.,1996b).

A associação inversa mais consistente que pode ser evidenciada dos estudos epidemiológicos, refere-se a um maior consumo de frutas e hortaliças, em especial as de cores verde-escuro e amarelo-alaranjado, ou níveis séricos elevados de $\beta$-caroteno, e baixas taxas de câncer de pulmão (Ziegler et al.,1992; Doll,1996; Ziegler et al.,1996b). Existem ainda relatos da existência de uma relação inversa entre a ingestão e/ou níveis séricos de vitamina $C$, vitamina E ou carotenóides, e proteção contra certos tipos de câncer (Block,1991; Knekt,1993; Flagg et al.,1995; Eichholzer et al.,1996; Ziegler et al.,1996a; Patterson et al.,1997).

De forma geral, os achados epidemiológicos motivaram instituições e pesquisadores a conduzir estudos clínico-epidemiológicos de intervenção, envolvendo a suplementação farmacológica com nutrientes denomi- nados antioxidantes. Considerando-se que estudos epidemiológicos observacionais não podem determinar se o efeito observado é devido especificamente a um nutriente ou grupo de nutrientes, os estudos de intervenção são necessários no sentido de esclarecer as relações causais sugeridas, como a relação entre $\beta$-caroteno e câncer de pulmão (Freudenheim,1993; Blumberg,1995; Ziegler et al.,1996b).

\section{Estudos epidemiológicos de intervenção - pre- venção primária}

Os estudos de quimioprevenção são conduzidos em fases I, II e III, por grandes instituições através de cooperação nacional e até mesmo internacional. Na fase I, estuda-se a toxicidade do agente em um pequeno grupo de indivíduos, identificando-se a dose máxima que oferece o mínimo de risco. A fase II corresponde a um estudo piloto com cerca de 100 a 1000 indivíduos, para definição operacional do projeto. Os estudos da fase III envolvem milhares de indivíduos adultos com aparência saudável, escolhidos aleatoriamente e em geral de populações de alto risco (acima de 40 anos), divididos em grupos tratado e placebo. Esses estudos são onerosos e complexos, pois encerram muitos anos de intervenção, tendo como alvo a incidência de câncer e/ou mortalidade (Freudenheim,1993; Alberts \& Garcia, 1995).

Na década de oitenta foram iniciados estudos de suplementação farmacológica com micronutrientes, coordenados pelo Instituto Nacional do Câncer $(\mathrm{NCl})$ dos EUA, e baseados em achados promissores de estudos epidemiológicos e de investigações em modelos animais (Mathews-Roth,1985; Bonne et al.,1990).

Atualmente, quatro estudos epidemiológicos de intervenção fase III já foram concluídos, e um estudo está em andamento. Dentre os estudos disponíveis, um apresentou resultado positivo, e outro, resultado nulo, sendo que nos dois restantes, constatou-se resultados negativos (Tabela 2).

No estudo realizado em Linxian (China ) (Blot et al.,1993), a suplementação conjunta de vitamina $E$, $\beta$-caroteno e selênio foi benéfica para indivíduos de populações com baixo consumo de micronutrientes e com concentrações plasmáticas reduzidas de carotenóides. Assim, observou-se uma redução de $21 \%$ na mortalidade por câncer de estômago e de $9 \%$ na mortalidade total.

No estudo Physicians' Health Study (PHS) (Hennekens et al.,1996), com 22071 médicos que foram acompanhados por 12 anos, não houve diferença significativa entre o grupo que recebeu suplementação de $\beta$-caroteno (50 mg em dias alternados) e o grupo placebo, em relação à incidência de câncer ou mortalidade por todas as causas.

No estudo Alpha-Tocopherol, Beta-Carotene Cancer Prevention Study (ATBC), os tabagistas filandeses 
Tabela 2. Estudos epidemiológicos de intervenção com as vitaminas A e E e $\beta$-caroteno ( $\beta c)$, na prevenção primária de câncer.

\begin{tabular}{|c|c|c|c|c|c|}
\hline $\begin{array}{c}\text { Estudo }^{1} \\
\text { (local, ano de } \\
\text { início, duração) }\end{array}$ & $\begin{array}{c}\text { Amostra } \\
(\mathrm{n}, \text { sexo, idade })\end{array}$ & Suplemento diário & Fator de risco & Efeito & Referência \\
\hline $\begin{array}{l}\text { Linxian Study } \\
\text { (China, 1986) } \\
5 \text { anos }\end{array}$ & $\begin{array}{l}29584 \mathrm{~m} / \mathrm{f}^{2} \\
40-69 \text { anos }\end{array}$ & $\begin{array}{c}15 \mathrm{mg} \beta \mathrm{c} \\
+30 \mathrm{mg} \text { vitamina } \mathrm{E} \\
+50 \mu \mathrm{g} \mathrm{Se}\end{array}$ & $\begin{array}{c}\text { População com alta } \\
\text { incidência de câncer de } \\
\text { esôfago e estômago e baixa } \\
\text { ingestão de micro-nutrientes }\end{array}$ & $\begin{array}{l}\text { Positivo ( } \Downarrow 21 \% \text { mortalidade } \\
\text { por câncer de estômago e } \Downarrow \\
9 \% \text { mortalidade total) }\end{array}$ & Blot et al. (1993) \\
\hline $\begin{array}{c}\mathrm{PHS}^{1} \\
(\mathrm{EUA}, 1992)\end{array}$ & $\begin{array}{c}22071 \mathrm{~m} \\
40-84 \text { anos }\end{array}$ & $25 \mathrm{mg} \beta \mathrm{c}$ & Nenhum & Nulo & Hennekens et al. (1996) \\
\hline 12 anos & & & & & \\
\hline $\begin{array}{c}\text { ATBC }^{1} \\
\text { (Finlândia, 1985) } \\
\text { 5-8 anos }\end{array}$ & $\begin{array}{c}29133 \mathrm{~m} \\
50-69 \text { anos }\end{array}$ & $\begin{array}{c}20 \mathrm{mg} \beta \mathrm{c} \text { ou } 50 \mathrm{mg} \text { de } \\
\text { vitamina } \mathrm{E} \text { ou } 20 \mathrm{mg} \beta \mathrm{c}+ \\
50 \mathrm{mg} \text { de vitamina } \mathrm{E}\end{array}$ & Tabagistas & $\begin{array}{l}\text { Negativo para } \beta c \text { ( } \Uparrow 18 \% \text { na } \\
\text { incidência de câncer de } \\
\text { pulmão e } \Uparrow 8 \% \text { na } \\
\text { mortalidade total) Nulo para } \\
\text { vitamina } E\end{array}$ & Alpha-tocopherol... (1994) \\
\hline $\begin{array}{c}\text { CARET }^{1} \\
\text { (EUA, 1991) } \\
4-5 \text { anos }\end{array}$ & $\begin{array}{l}18134 \mathrm{~m} / \mathrm{f} \\
50-69 \text { anos }\end{array}$ & $\begin{array}{c}30 \mathrm{mg} \beta \mathrm{c}+25000 \mathrm{Ul}^{3} \mathrm{de} \\
\text { vitamina } \mathrm{A}\end{array}$ & $\begin{array}{c}\text { Tabagistas ou trabalhadores } \\
\text { expostos ao asbesto }\end{array}$ & $\begin{array}{l}\text { Negativo ( } \Uparrow 28 \% \text { na } \\
\text { incidência de câncer de } \\
\text { pulmão e } \Uparrow 17 \% \text { na } \\
\text { mortalidade total) }\end{array}$ & Omenn et al. (1996) \\
\hline $\begin{array}{c}\text { SUVIMAX } \\
\text { (França, 1994) } \\
8 \text { anos (duração } \\
\text { prevista) }\end{array}$ & $\begin{array}{l}12749 \mathrm{~m} / \mathrm{f} \\
35-60 \text { anos }\end{array}$ & $\begin{array}{l}6 \mathrm{mg} \beta c(1000 \mathrm{ER})^{4}+30 \\
\text { mg de vitamina } E+120 \mathrm{mg} \\
\text { de vitamina } C+20 \mathrm{mg} \text { de } \\
\text { zinco }+100 \mu \mathrm{g} \text { de selênio }\end{array}$ & Nenhum & Em andamento & Hercberg et al. (1998) \\
\hline
\end{tabular}

(1) PHS = Physicians' Health Study; ATBC = Alpha-Tocopherol, Beta-carotene Cancer Prevention Study; CARET = Beta-carotene Retinol Efficacy Trial; SUVIMAX = Suplementação com Vitaminas e Minerais Antioxidantes.

${ }^{(2)} \mathrm{m}=$ masculino; $\mathrm{f}=$ feminino.

${ }^{(3)} \mathrm{UI}=$ Unidades Internacionais (1 UI equivale a 0,3 $\mu$ g de retinol).

${ }^{(4)} \mathrm{ER}=$ equivalente de retinol ( $1 \mu \mathrm{g}$ de retinol ou $6 \mu \mathrm{g}$ de $\beta$-caroteno).

que receberam $\beta$-caroteno $(20 \mathrm{mg} / \mathrm{dia}$ ) e $\beta$-caroteno mais vitamina $E(50 \mathrm{mg} /$ dia) não responderam positivamente à suplementação; ao contrário, constatou-se nesses indivíduos um aumento de $18 \%$ na incidência de câncer de pulmão e de $8 \%$ na mortalidade total (Alpha-Tocopherol..., 1994; Albanes et al.,1995). Entretanto, observou-se em todos os grupos, que o consumo e os níveis séricos de $\beta$-caroteno e de $\alpha$-tocoferol ao início do estudo foram inversamente associados com o risco de câncer de pulmão no decorrer do estudo (Alpha-Tocopherol..., 1994), e no grupo placebo, que a concentração de $\beta$-caroteno no plasma foi inversamente proporcional à incidência de câncer de pulmão (Albanes et al.,1995), achados consistentes com aqueles dos estudos observacionais (Knekt,1993; Ziegler et al.,1996a; Ziegler et al.,1996b). O grupo suplementado com $\alpha$-tocoferol (50 $\mathrm{mg} / \mathrm{dia})$ não apresentou nenhuma diferença na incidência de câncer de pulmão em relação ao grupo controle.

O estudo Beta-Carotene Retinol Efficacy Trial (CARET), por sua vez, investigou o efeito da suplementação conjunta de $\beta$-caroteno ( $30 \mathrm{mg} /$ dia) e vitamina A (25 $000 \mathrm{U}$ ) em uma população de alto risco para câncer de pulmão (fumantes e trabalhadores expostos ao asbesto), por um período de cerca de 5 anos (Omenn et al.,1996). Este estudo foi interrompido 21 meses antes do término, uma vez que foi constatado, no grupo suplementado, um aumento na incidência de morte por qualquer causa, incluindo câncer de pulmão (Tabela 2).

De uma forma geral, estes achados inesperados levaram a comunidade científica a reconsiderar a relação entre antioxidantes e câncer, à luz da influência de fatores tais como dose suplementada, fator de risco, idade dos indivíduos, etc.

Neste contexto foi proposto o estudo Suplementação com Vitaminas e Minerais Antioxidantes (SUVIMAX) iniciado em 1994, na França (Hercberg et al.,1998). Trata-se de um estudo epidemiológico prospectivo, de intervenção, que tem como objetivo avaliar os efeitos do consumo concomitante de vitaminas e minerais antioxidantes, suplementados em doses mais fisiológicas 
(próximas às recomendações nutricionais) sobre a mortalidade por câncer, por um período de 8 anos. A população do referido estudo constitui-se de indivíduos não submetidos a fatores de risco e mais jovens que aquela dos estudos mencionados anteriormente (Tabela 2). O grande envolvimento dos participantes, bem como alguns resultados preliminares indicam que esse estudo pode contribuir efetivamente para um maior entendimento sobre a relação entre nutrientes antioxidantes e o câncer (Preziosi et al.,1998).

\section{DISCUSSÃO}

Várias hipóteses têm sido levantadas na tentativa de explicar os efeitos negativos da suplementação com $\beta$-caroteno (isolado ou associado à vitamina $E$ ), no estudo ATBC, bem como do carotenóide administrado em conjunto com a vitamina $A$, no estudo CARET, na prevenção primária de câncer.

Discute-se que os indivíduos, ao início dos estudos ATBC e CARET, estavam com idade "avançada" e apresentavam história de exposição prolongada à fumaça do tabaco ou ao asbesto, provavelmente se encontrando em fase adiantada do processo de promoção da carcinogênese (De Luca \& Ross,1996; Burri,1997). Desse modo, o $\beta$-caroteno poderia estar sendo administrado tardiamente no processo, uma vez que o carotenóide parece ser efetivo quando suplementado antes ou durante os estágios iniciais da carcinogênese (Moreno et al.,1991; Rizzi et al.,1997). Além disso, quando administrado em excesso ou em quantidades bem acima daquelas normalmente ingeridas pelas populações saudáveis, essa substância pode agir como pró-oxidante, ocasionando efeitos tóxicos, inclusive potencializando o estado oxidativo crônico presente nos pulmões de tabagistas inveterados (Naves \& Moreno,1998; Palozza,1998). Vale acrescentar que as doses administradas do carotenóide (Tabela 2) representavam no mínimo dez vezes o consumo habitual dos indivíduos estudados.

Contudo, relatos de estudos epidemiológicos observacionais e de estudos em modelos animais indicam que o $\beta$-caroteno pode exercer efeito protetor contra o câncer, em populações saudáveis, e em combinação com substâncias antioxidantes, se ingerido em quantidades mais fisiológicas, conforme encontrado em uma dieta rica em frutas e hortaliças (Block \& Langseth,1994; Naves,1998). A combinação entre ácido ascórbico, $\alpha$-tocoferol e $\beta$-caroteno torna-se mais efetiva, visto que esses nutrientes podem interagir no ambiente celular e potencializar a defesa antioxidante (Niki et al.,1995) resultando, por exemplo, em proteção contra dano oxidativo no DNA (Anderson,1996; Cozzi et al.,1997; Pool-Zobel et al.,1997). A ação sinérgica destas substâncias antioxidantes na quimioprevenção de câncer já foi demonstrada em modelo in vivo de carcinogênese (Shklar et al.,1993).
Sendo assim, os antioxidantes parecem atuar como substâncias quimiopreventivas quando consumidos em conjunto e em doses mais fisiológicas, hipótese a ser confirmada através do estudo SUVIMAX (Tabela 2).

Vale acrescentar que os antioxidantes são primariamente, moduladores fisiológicos, conforme proposto por Olson (1996) e, portanto, podem influenciar outros processos no organismo além do balanço oxidativo. Assim, a atividade antioxidante desses compostos não explica todos os efeitos biológicos observados, particularmente para carotenóides, que parecem atuar de forma intrínseca, por exemplo, nas comunicações intercelulares via junções comunicantes, através da regulação da expressão do gene da conexina 43, uma proteína estrutural dessas junções (Naves \& Moreno, 2000).

\section{RECOMENDAÇÕES}

Os resultados dos estudos epidemiológicos de intervenção não indicam que o uso de suplementos de micronutrientes pode reduzir as taxas de incidência de câncer. Por outro lado, organizações de diferentes países têm publicado guias alimentares que estabelecem algumas recomendações básicas, dentre elas a adoção de uma dieta rica em frutas e hortaliças (Bruce,1987; Willett,1994; Hunt,1996). Recomenda-se, mais especificamente, o consumo de 5 ou mais porções diárias desses alimentos (Havas et al.,1994), sendo que a Organización Mundial de La Salud sugere um consumo diário mínimo de $400 \mathrm{~g}$ de frutas e hortaliças, como uma estratégia de prevenção de doenças crônicas, especialmente o câncer. Vale lembrar que a prática dessas recomendações resulta em uma dieta mais equilibrada e saudável, gerando sobretudo aumento no aporte de vitamina $C$, carotenóides e de fibras alimentares, bem como redução no consumo de energia e gorduras (Organización Mundial...,1990; Cox et al.,1998).

A suplementação de vitaminas e minerais, por sua vez, está preconizada somente no caso de aporte insuficiente na alimentação, não excedendo às quantidades recomendadas pelo Conselho Nacional de Pesquisa dos EUA - Recommended Dietary Allowances (RDA) - (Silva \& Naves, 1998) e em certas situações fisiológicas e em situações clínicas específicas (Hunt,1996; Zeisel, 2000). Entretanto, a ingestão de quantidades um pouco acima daquelas recomendadas, através do consumo de uma alimentação variada, rica em frutas e hortaliças, parece ser bastante segura e saudável (Organización Mundial...,1990; Willett,1994; Hathcock, 1997).

No caso da vitamina $C$, dados disponíveis indicam que a ingestão de 80 a $120 \mathrm{mg} /$ dia pode reduzir o risco de doenças crônicas não-infecciosas, incluindo o câncer, e que fumantes necessitam de um aporte mais elevado, de até $140 \mathrm{mg} / \mathrm{dia}$ (Weber et al.,1996). Blumberg (1995) sugere o consumo de cerca de $150 \mathrm{mg}$, ou seja, $21 / 2$ vezes a dose diária recomendada para indivíduos adultos de acordo com a RDA (Silva \& Naves,1998), para se alcançar 
concentrações plasmáticas de vitamina $C$ associadas com um menor risco de doenças crônicas. Ao contrário, o consumo de vitamina $\mathrm{C}$ em doses mais elevadas pode ser deletério para o organismo, conforme observado através do aumento de lesões potencialmente mutagênicas em indivíduos saudáveis suplementados com 500 mg/dia durante 6 meses (Podmore et al., 1998).

$A$ ingestão de vitamina $E$, na quantidade de 30 mg/dia, estabelecida no estudo SUVIMAX (Tabela 2) e que corresponde a cerca de 3 vezes a quantidade recomendada (Silva \& Naves, 1998) parece ser segura e eficaz na proteção contra doenças crônicas (Blumberg,1995; Hercberg et al., 1998).

Em relação aos carotenóides, os relatos de estudos epidemiológicos observacionais sugerem que a ingestão de cerca de $4 \mathrm{mg} /$ dia de carotenóides, quantidade presente em uma alimentação rica em frutas e hortaliças, pode proteger contra o câncer sem apresentar riscos à saúde (Ziegler et al.,1996a; Naves,1998). Recomenda-se ainda que a ingestão de $\beta$-caroteno (e demais carotenóides) não exceda a $10 \mathrm{mg} / \mathrm{dia}$, especialmente no caso de fumantes (Burri, 1997).

\section{CONCLUSÃO}

Os resultados de estudos epidemiológicos indicam que a ingestão de quantidades fisiológicas de antioxidantes, tais como as vitaminas $\mathrm{C}$ e $\mathrm{E}$ e os carotenóides, pode retardar ou prevenir o aparecimento de câncer. Assim, o consumo de uma dieta rica em frutas e hortaliças, contendo quantidades dessas substâncias próximas às recomendadas nutricionalmente, contribui com a defesa antioxidante do organismo, inibindo danos oxidativos em macromoléculas.

$\mathrm{O}$ aporte de quantidades mais elevadas, na forma de suplementos, não está indicado, podendo inclusive ser deletério para o organismo, conforme constatado nos estudos de suplementação de $\beta$-caroteno para fumantes. São necessárias mais informações científicas para comprovar os eventuais benefícios da suplementação com nutrientes antioxidantes. O estudo SUVIMAX, em andamento na França, poderá contribuir significativamente para o esclarecimento desta questão.

\section{REFERÊNCIAS BIBLIOGRÁFICAS}

ALBANES, D., HEINONEN, O.P., HUTTUNEN, J.K., TAYLOR, P.R., VIRTAMO, J., EDWARDS, B.K., HAAPAKOSKI, J., RAUTALAHTI, M., HARTMAN, A.M., PALMGREN, J., GREENWALD, P. Effects of a-tocopherol and $\beta$-carotene supplements on cancer incidence in the alpha-tocopherol beta-carotene cancer prevention study. American Journal of Clinical Nutrition, Bethesda, v.62, p.1427S-1430S,1995. Supplement 6.

ALBERTS, D.S., GARCIA, D.J. An overview of clinical cancer chemoprevention studies with emphasis on positive phase
III studies. Journal of Nutrition, Philadelphia, v.125, p.692S-697S,1995. Supplement 3.

ALPHA-TOCOPHEROL, Beta-Carotene Cancer Prevention Study Group: the effect of vitamin $E$ and beta carotene on the incidence of lung cancer and other cancers in males smokers. New England Journal of Medicine, Boston, v.330, n.15, p.1029-1035, 1994.

ANDERSON, D. Antioxidant defences against reactive oxygen species causing genetic and other damage. Mutation Research, Amsterdam, v.350, n.1, p.103-108,1996.

AZOUBEL, L.M.O., GARCIA, R.W.D., NAVES, M.M.V. Tabela de composição de alimentos. In: DUTRA-DE-OLIVEIRA, J.E., MARCHINI, J.S. Ciências nutricionais. São Paulo : Sarvier, 1998. p.363-376.

BIANCHI, M.L.P., ANTUNES, L.M.G. Radicais livres e os principais antioxidantes da dieta. Revista de Nutrição, Campinas, v.12, n.2, p.123-130, 1999.

BLOCK, G. Vitamin C and cancer prevention: the epidemiologic evidence. American Journal of Clinical Nutrition, Bethesda, v.53, p.270S-282S, 1991. Supplement 1.

BLOCK, G., LANGSETH, L. Antioxidant vitamins and disease prevention. Food Technology, Chicago, v.48, n.7, p.80-84, 1994.

BLOT, W.J., LI, J.Y., TAYLOR, P.R., GUOW., DAWSEY, S., WANG, G.Q., YANG C.S., ZHENG, S.F., GAIL, M., LI, G.Y., YU,Y., LIU, B.Q., TANGREA, J., SUN, Y.H., LIU, F., FRAUMENI JR., J.F., ZHANG, Y.H., LI, B. Nutrition intervention trials in Linxian, China: suplementation with specific vitamin/mineral combinations, cancer incidence, and disease-specific mortality in the general population. Journal of the National Cancer Institute, Bethesda, v.85, n.18, p.1483-1491, 1993.

BLUMBERG, J.B. Considerations of the scientific substantiation for antioxidant vitamins and $\beta$-carotene in disease prevention. American Journal of Clinical Nutrition, Bethesda, v.62, p.1521S-1526S,1995. Supplement 6.

BONNE, C.W., KELLOFF, G.J., MALONE, W.E. Identification of candidate cancer chemopreventive agents and their evaluation in animal models and human clinical trials: a review. Cancer Research, Philadelphia, v.50, n.1, p.2-9, 1990.

BRUCE, A. Dietary recommendations in cancer prevention. Annals of Clinical Research, Helsinki, v.19, n.5, p.313-320, 1987.

BURRI, B.J. Beta-carotene and human health: a review of current research. Nutrition Research, New York, v.17, n.3, p.547-580, 1997.

BURTON, G.W., INGOLD, K.U. $\beta$-Carotene: an unusual type of lipid antioxidant. Science, Washington DC, v.224, n.4649, p.569-573, 1984.

BYERS, T., PERRY, G. Dietary carotenes, vitamin C and vitamin E as protective antioxidants in human cancer. Annual Review of Nutrition, Palo Alto, v.12, p.139-159, 1992.

CARAGAY, A.B. Cancer-preventive foods and ingredients. Food Technology, Chicago, v.46, n.4, p.65-68, 1992.

CERUTTI, P.A. Oxy-radicals and cancer. Lancet, London, v.344, n.8926, p.862-863, 1994.

COX, D.N., ANDERSON, A.S., REYNOLDS, J., MCKELLAR, S., LEAN, M.E.J., MELA, D.J. Take five, a nutrition education intervention to increase fruit and vegetable intakes: impact on 
consumer choice and nutrient intakes. British Journal of Nutrition, London, v.80, p.123-131, 1998.

COZZI, R., RICORDY, R., AGLITTI, T., GATTA, V., PERTICONE, P., De SALVIA, R. Ascorbic acid and $\beta$-carotene as modulators of oxidative damage. Carcinogenesis, London, v.18, n.1, p.223-228, 1997.

DE LUCA, L. M., ROSS, S. A. Beta-carotene increases lung cancer incidence in cigarette smokers. Nutrition Reviews, New York, v.54, n.6, p.178-180, 1996.

DOLL, R. Nature and nurture: possibilities for cancer control. Carcinogenesis, London, v.17, n.2, p.177-184, 1996.

EICHHOLZER, M., STÄHELIN, H.B., GEY, K.F., LUDIN, E., BERNASCONI, F. Prediction of male cancer mortality by plasma levels of interacting vitamins:17-year follow-up of the prospective Basel study. International Journal of Cancer, Geneva, v.66, n.2, p.145-150, 1996.

FLAGG, E.W., COATES, R.J., GREENBERG, R. S. Epidemiologic studies of antioxidants and cancer in humans. Journal of the American College of Nutrition, New York, v.14, n.5, p.419-427, 1995.

FREUDENHEIM, JO.L. A review of study designs and methods of dietary assessment in nutritional epidemiology of chronic disease. Journal of Nutrition, Philadelphia, v.123, n.2, p.401-405, 1993.

GODOY, H.T., RODRIGUEZ-AMAYA, D.B. Occurrence of cis-isomers of provitamin A in Brazilian fruits. Journal of Agricultural and Food Chemistry, Washington, v.42, n.6, p.1306-1313, 1994.

HATHCOCK, J.N. Vitamins and minerals: efficacy and safety. American Journal of Clinical Nutrition, Bethesda, v.66, n.2, p.427-437, 1997.

HAVAS, S., HEIMENDINGER, J., REYNOLDS, K., BARANOWSKI, T., NICKLAS, T.A., BISHOP, D., BULLER, D., SORENSEN, G., BERESFORD, S.A.A., COWAN, A., DAMRON, D. A day for better health: a new research initiative. Journal of the American Dietetic Association, Chicago, v.94, n.1, p.32-36, 1994.

HENNEKENS, C.H., BURING, J.E., MANSON, J.E., STAMPFER, M., ROSNER, B., COOK, N.R., BELANGER, C., LAMOTTE, F., GAZIANO, J.M., RIDKER, P.M., WILLETT, W., PETO, R. Lack of effect of long-term supplementation with beta carotene on the incidence malignant neoplasms and cardiovascular disease. New England Journal of Medicine, London, v.334, n.18, p.1145-1149, 1996.

HERBERT,V. Prooxidant effects of antioxidant vitamins. Journal of Nutrition, Philadelphia, v.126, p.1197S-1200S, 1996. Supplement 4.

HERCBERG, S., GALAN, P., PREZIOSI, P., ROUSSEL, A.M., ARNAUD, J., RICHARD, M.J., MALVY, D., PAUL-DAUPHIN, A., BRIANÇON, S., FAVIER, A. Background and rationale behind the SUVIMAX study, a prevention trial using nutritional doses of a combination of antioxidant vitamins and minerals to reduce cardiovascular diseases and cancers. International Journal for Vitamin and Nutrition Research, Bern, v.68, n.1, p.3-20, 1998.

HUNT, J.R. Position of the American Dietetic Association: vitamin and mineral supplementation. Journal of the American Dietetic Association, Chicago, v.96, n.1, p.73-77, 1996.

KNEKT, P. Epidemiology of vitamin E: evidence for anticancer effects in humans. In: PACKER, L., FUNCHS, J. (Ed.). Vitamin
$E$ in health and disease. New York : Marcel Dekker, 1993. p.141-164.

KUHN, R.E., GUZMÁN-SILVA, M.A., GUIMARÃES, J.S.P. Dialquilnitrosaminas e câncer. Revista Brasileira de Cancerologia, Rio de Janeiro, v.37, n.1/4, p.19-26, 1991.

LOTAN, R. Retinoids in cancer chemoprevention. FASEB Journal, Bethesda, v.10, n.9, p.1031-1039, 1996.

MANGELS, A.R., HOLDEN, J.M., BEECHER, G.R., FORMAN, M.R., LANZA, E. Carotenoid content of fruits and vegetables: an evolution of analytic data. Journal of the American Dietetic Association, Chicago, v.93, n.3, p.284-296, 1993.

MATHEWS-ROTH, M.M. Carotenoids and cancer prevention: experimental and epidemiological studies. Pure and Applied Chemistry, London, v.57, n.5, p.717-722, 1985.

MORENO, F.S., RIZZI, M.B.S.L., DAGLI, M.L.Z., PENTEADO, M.C.V. Inhibitory effects of $\beta$-carotene on preneoplastic lesions induced in Wistar rats by the resistant hepatocyte model. Carcinogenesis, London, v.12, n.10, p.1817-1822, 1991.

NAVES, M.M.V. Beta-caroteno e câncer. Revista de Nutrição, Campinas, v.11, n.2, p.99-115, 1998.

NAVES, M.M.V., MORENO, F.S. $\beta$-Carotene and cancer chemoprevention: from epidemiological associations to cellular mechanisms of action. Nutrition Research, New York, v.18, n.10, p.1807-1824, 1998.

NAVES, M.M.V., MORENO, F.S. Comunicações intercelulares com conexinas: importância na carcinogênese e papel modulador dos carotenóides. Revista Brasileira de Ciências Farmacêuticas, São Paulo, v.36, n.1, p.1-11, 2000.

NIKI, E. $\alpha$-Tocopherol. In: CADENAS, E., PACKER, L. (Ed.). Handbook of antioxidants. New York : Marcel Dekker, 1996. p.3-25.

NIKI, E., NOGUCHI, N., TSUCHIHASHI, H., GOTOH, N. Interaction among vitamin $\mathrm{C}$, vitamin $\mathrm{E}$, and $\beta$-carotene. American Journal of Clinical Nutrition, Bethesda, v.62, p.1322S-1326S, 1995. Supplement 6.

OLSON, J.A. Provitamin A function of carotenoids: the conversion of $\beta$-carotene into vitamin A. Journal of Nutrition, Philadelphia, v.119, n.1, p.105-108, 1989.

OLSON, J.A. Vitamins: the tortuous path from needs to fantasies. Journal of Nutrition, Philadelphia, v.124, p.1771S-1776S, 1994.

OLSON, J.A. Benefits and liabilities of vitamin A and carotenoids. Journal of Nutrition, Philadelphia, v.126, p.1208S-1212S, 1996. Supplement 4.

OMENN, G.S., GOODMAN, G.E., THORNQUIST, M.D., BALMES, J., CULLEN, M.R., GLASS, A., KEOGH, J.P., MEYSKENS JR., F.L., VALANIS, B., WILLIAMS JR., J.H., BARNHART, S., CHERNIACK, M.G., BRODKIN, C.A., HAMMAR, S. Risk factors for lung cancer and for intervention effects in CARET, the beta-carotene and retinol efficacy trial. Journal of the National Cancer Institute, Bethesda, v.88, n.21, p.1550-1558, 1996.

ORGANIZACIÓN MUNDIAL DE LA SALUD. Dieta, nutrición y prevención de enfermedades crónicas. Ginebra, 1990. p.97-132. (Série de Informes Técnicos, 797).

PALOZZA, P. Prooxidant actions of carotenoids in biologic systems. Nutrition Reviews, New York, v.56, n.9, p.257-265, 1998. 
PATTERSON, R.E., WHITE, E., KRISTAL, A.R., NEUHOUSER, M.L., POTTER, J.D. Vitamin supplements and cancer risk: the epidemiologic evidence. Cancer: Causes and Control, London, v.8, n.5, p.786-802, 1997.

PAUL, A.A., SOUTHGATE, D.A.T. McCance and Widdowson's the composition of foods. 4.ed. Amsterdam : Elsevier-North, 1978.

PINHEIRO, A.B.V., LACERDA, E.M.A., BENZECRY, E.H., GOMES, M.C.S., COSTA, V.M. Tabela para avaliação de consumo alimentar em medidas caseiras. 4.ed. São Paulo : Atheneu, 2001.

PITOT, H.C., DRAGAN, Y.P. Chemical carcinogenesis. In: KLAASSEN, C.D. (Ed.). Casarett and Doull's toxicology: the basic science of poisons. 5.ed. New York : McGraw-Hill, 1996. p.201-267.

PODMORE, I.D., GRIFFITHS, H.R., HERBERT, K.E., MISTRY, N., MISTRY, P., LUNEC, J. Vitamin $C$ exhibits pro-oxidant properties. Nature, London, v.392, n.6676, p.559, 1998.

POOL-ZOBEL, B.L., BUB, A., MÜLLER, H., WOLLOWSKI, I., RECHKEMMER, G. Consumption of vegetables reduces genetic damage in humans: first results of a human intervention trial with carotenoid-rich foods. Carcinogenesis, London, v.18, n.9, p.1847-1850, 1997.

PREZIOSI, P., GALAN, P., HERBETH, B., VALEIX, P., ROUSSEL, A.M., MALVY, D., PAUL-DAUPHIN, A., ARNAUD, J., RICHARD, M.J., BRIANCON, S., FAVIER, A., HERCBERG, S. Effects of supplementation with a combination of antioxidant vitamins and trace elements, at nutritional doses, on biochemical indicators markers of the antioxidant system in adult subjects. Journal of the American College of Nutrition, New York, v.17, n.3, p.244-249, 1998.

RIZZI, M.B.S.L., DAGLI, M.L.Z., JORDÃO JR., A.A., PENTEADO, M.V.C., MORENO, F.S. $\beta$-Carotene inhibits persistent and stimulates remodeling $\gamma \mathrm{GT}$-positive preneoplastic lesions during early promotion of hepatocarcinogenesis. International Journal for Vitamin and Nutrition Research, Bern, v.67, n.6, p.415-422, 1997.

ROCK, C.L., JACOB, R.A., BOWEN, P.E. Update on the biological characteristics of the antioxidant micronutrients: vitamin $C$, vitamin E, and the carotenoids. Journal of the American Dietetic Association, Chicago, v.96, p.693-702, 1996.

SHKLAR, G., SCHWARTZ, J., TRICKLER,D., CHEVERIE,S.R. The effectiveness of a mixture of $\beta$-carotene, $\alpha$-tocopherol, glutathione, and ascorbic acid for cancer prevention. Nutrition and Cancer, Philadelphia, v.20, n.2, p.145-151, 1993.
SILVA, M.R., NAVES, M.M.V. (Org.) Manual de nutrição e dietética. 2.ed. rev. atual. Goiânia : UFG, 1998. cap. 2: Recomendações de energia e nutrientes, p. 21-34.

SPORN, M.B., ROBERTS, A.B. What is a retinoid? Ciba Foundation Symposium, London, v.113, p.1-5, 1985.

STAHL, W., SIES, H. Antioxidant defense: vitamins E and C and carotenoids. Diabetes, New York, v.46, n.5, p.14S-18S,1997. Supplement 2.

TAVANI, A., LA VECCHIA, C. Fruit and vegetable consumption and cancer risk in mediterranean population. American Journal of Clinical Nutrition, Bethesda, v.61, p.1374S-1377S, 1995. Supplement 6 .

Van POPPEL, G., Van den BERG, H. Vitamins and cancer. Cancer Letters, Shannon, v.114, p.195-202, 1997.

WEBER, P., BENDICH, A., SCHALCH, W. Vitamin $\mathrm{C}$ and human health: a review of recent data relevant to human requirements. International Journal for Vitamin and Nutrition Research, Bern, v.66, n.1, p.19-30, 1996.

WHITNEY, E.N., ROLFES, S.R. Understanding nutrition. 8.ed. Belmont : ITP, 1999. Appendix H: Table of food composition.

WILLETT, W.C. Diet and health: what should we eat? Science, Washington DC, v.264, p.532-537, April 22, 1994.

ZEISEL, S.H. Is there a metabolic basis for dietary supplementation? American Journal of Clinical Nutrition, Bethesda, v.72, p.507S-511S, 2000. Supplement 2.

ZIEGLER, R.G., SUBAR, A.F., CRAFT, N.E., URSIN, G., PATTERSON, B.H., GRAUBARD, B.I. Does $\beta$-carotene explain why reduced cancer risk is associated with vegetable and fruit intake? Cancer Research, Philadelphia, v.52, p.2060S-2066S, 1992. Supplement 7.

ZIEGLER, R.G., COLAVITO, E.A., HARTGE, P., MCADAMS, M.J., SCHOENBERG, J.B., MASON, T.J., FRAUMENI JR., J.F. Importance of $\alpha$-carotene, $\beta$-carotene, and other phytochemicals in the etiology of lung cancer. Journal of the National Cancer Institute, Bethesda, v.88, n.9, p.612-615, 1996a.

ZIEGLER, R.G., MAYNE, S.T., SWANSON, C.A. Nutrition and lung cancer. Cancer Causes and Control, London, v.7, n.1, p.157-177, 1996b.

Recebido para publicação em 14 de junho e aceito em 1 de setembro de 2000 . 